Dominique Méda ${ }^{1}$

\title{
Centralité du travail, plein emploi de qualité et développement humain
}

Nous sommes sans doute aujourd'hui au plus au point des sociétés fondées sur le travail. Mais c'est là le résultat d'une histoire et d'une construction, et non un fait de nature. Nous vivons avec une idée " moderne " du travail qui s'est construite au cours des siècles, chacun rajoutant une “ couche” de signification différente des précédentes. Il n’y a pas une catégorie de travail, une essence du travail qui aurait perduré identiquement au travers des siècles imperturbablement dotée des mêmes significations et aucun concept n'a plus varié à travers l'histoire, sauf à ne le penser que comme la simple présence “opposante ” de l'homme dans le monde.

Il faudra attendre le $19^{\text {ème }}$ siècle pour voir le travail chargé des multiples fonctions que lui assigne par exemple Marie Jahoda. Le travail, outre sa fonction manifeste - apporter un revenu -, remplirait selon cet auteur cinq fonctions indispensables : “il impose une structure temporelle de la vie ; il crée des contacts sociaux en dehors de la famille ; il donne des buts dépassant les visées propres ; il définit l’identité sociale et il force à l'action ”. Il faudra aussi attendre cette époque pour que se développe la croyance, quasi mythique, selon laquelle le travail constitue pour l'homme l'une des plus hautes formes d'expression.

Il ne s'agit pas de soutenir que le travail n'aurait pas existé à un moment puis aurait brutalement fait son apparition, mais bien plutôt que, si les hommes ont toujours dû se confronter à la nature pour survivre et ont toujours transformé leurs conditions de vie, ces activités, d'une part, n'ont pas toujours été rassemblées sous une catégorie unique et, d'autre part, n'étaient pas au fondement de l'ordre social. Comprendre le concept de travail comme le résultat d'une construction mais aussi d'une certaine illusion devrait nous permettre aujourd'hui, non seulement de le reconnaître comme un bien premier fondamental auquel tout individu doit avoir accès, mais aussi de comprendre qu'il ne peut pas épuiser l'ensemble des manières pour l'être humain de s'exprimer ou de mettre le monde en valeur.

Ce qui milite en faveur de la substitution d'une philosophie de l'activité à une philosophie du travail ou de la centralité du travail. C'est certainement la voie la plus prometteuse pour obtenir un " plein emploi de qualité " capable de fonder, pour les hommes et les femmes, une véritable “conciliation” des différentes activités qui leur incombent, au-delà d'une division sexuelle des rôles devenue anachronique.(...)

Nous vivons donc aujourd'hui avec un concept du travail qui est un “ monstre ”, le produit de la juxtaposition et de l'assemblage non repensés de ces trois dimensions du travail : le travail comme facteur de production, comme essence de l'homme et comme système de distribution des revenus, des droits et des protections. Les contradictions entre ces trois définitions sont légion. Cet héritage non " retravaillé " explique que nous ne parvenions pas à nous accorder

\footnotetext{
${ }^{\mathbf{1}}$ Extraits d'un article publié dans Cités, $\mathrm{n}^{\circ}$ 8, 2001

Auteur de Le Travail. Une valeur en voie de disparition, “Alto ”, Aubier, 1995, rééd. Champs-Flammarion, 1998 ; Qu'est-ce que la richesse ?, “ Alto ”, Aubier, 1999, rééd. Champs-Flammarion, 2000 ; Le temps des femmes. Pour un nouveau partage des rôles, Flammarion, 2001.
} 
sur une définition simple du travail, ni à choisir entre une définition “ extensive " : le travail comme activité qui permet à l'homme, outre d'obtenir un revenu, de transformer le monde, d'avoir des contacts avec l'extérieur, d'entrer dans des communautés autres que familiales, de s'exprimer...et une définition plus “ réduite” : le travail comme participation rémunérée à la production de biens et services. (...)

\section{$\underline{\text { L'illusion du travail comme vocation exclusive de l'homme }}$}

[C'est pour cette raison qu']il me semble nécessaire de préférer désormais à une philosophie du travail (toujours pensée comme de la centralité du travail) une philosophie de l'activité, partant de l'activité humaine comme genre qui admet des espèces irréductiblement différentes : l'activité de production - le travail -, qui permet à chacun de participer à la production rémunérée de biens et services, d'avoir une place dans la société d’obtenir un certain type d'utilité et de reconnaissance sociale ; l'activité politique, qui permet (ou devrait permettre) à chacun de participer à la discussion, à l'élection, au débat, au choix des conditions de vie communes ; l'activité amicale, amicale, parentale, familiale, qui permet de développer des types de liens et des actions qui n’ont rien à voir avec le travail ; les activités personnelles visant à se former ou à se développer librement...) ; l'ideal d'une bonne société étant de garantir à chaque individu l'accès à la gamme entière de ces activités, toutes nécessaires au bien-être individuel et social.

Un tel raisonnement ne vise donc en rien à la disparition du travail : il se situe délibérement du côté du souhaitable, pour s’interroger sur les fins de la société, concevant celles-ci comme plurielles et préférant au seul objectif d'augmentation de la production celui de développement ou de civilisation, et en déduisant, plutôt que la centralité du travail ou de la production, un développement équilibré des différentes activités nécessaires au bien-être individuel et social ${ }^{2}$ et, derechef la nécessité de règles permettant d'organiser cette coexistence, la poursuite par les individus de cette pluralité d'objectifs. Circonscrire la place du travail, intégrer de manière bien plus approfondie qu'aujourd'hui le travail et le reste de la vie, et les différents temps sociaux, revoir en profondeur les investissements temporels déséquilibrés que consacrent aujourd'hui hommes et femmes au travail, à l'éducation, aux soins, aux tâches domestiques, aux activités sociales et aux loisirs, est sans doute aujourd'hui la meilleure façon de parvenir, en même temps, à rendre le travail plus humain, le rapport au travail des différentes catégories sociales moins hétérogène et la société, finalement, plus riche.

\section{Qu'est-ce qu'un plein emploi de qualité : un emploi intégré dans la vie individuelle et sociale}

Il faut sans doute ici s'arrêter un instant sur le contexte nouveau dans lequel cette recherche de compatibilité s'inscrit aujourd'hui : il est en effet radicalement différent du contexte “beveridgien" de l'après-guerre ${ }^{3}$, non seulement parce que les instruments dont l'Etat disposait pour tenir ensemble toutes les "manettes" permettant d'obtenir le plein emploi n’existent plus, parce que le processus de mondialisation, même remis en cause, a changé la donne en profondeur, parce que l'emploi est devenu plus flexible et aussi parce qu'il ne s'agit

\footnotetext{
${ }^{2}$ et notamment la substitution d'indicateurs pluriels tels que ceux présentés dans la série des Rapports sur le développement humain du PNUD à l'utilisation du seul PIB, cf. D. Méda, Qu'est-ce que la richesse ?,op.cit ${ }^{3}$ cf D. Méda, “Le plein emploi de qualité, une figure du bien commun? ?, L‘Economie politique, septembre 2000
} 
plus du plein emploi du travailleur masculin mais bien de celui de tous les hommes et les femmes d'une société.

Si les contraintes temporelles sont ressenties de manière aussi fortes par les individus, c'est en effet non seulement parce que les contraintes de flexibilité issues du système productif se sont accrues mais aussi parce que l'organisation sociale sur laquelle ces contraintes s'exercent s'est considérablement transformée. Parmi ces transformations, une doit retenir en priorité notre attention: la considérable augmentation, ces trente dernières années, de l'activité féminine (le taux d'activité des femmes de 25 à 49 ans s'élève aujourd'hui à $80 \%$ alors qu'il était de $41,5 \%$ en 1962), et de ses modalités, puisque la majeure partie des femmes n'interrompent désormais plus leur activité au moment où elles ont des enfants, à la différence de la situation qui prévalait antérieurement.

Autrement dit, la société est composée de plus en plus de couples bi-travailleurs ou de familles monoparentales chargées d'enfants, ce qui signifie que la spécialisation qui prévalait dans les années soixante, l'homme travaillant et externalisant l'ensemble des tâches domestiques et familiales sur la femme, véritable "réservoir de temps "(ce qui signifie la gestion non seulement d'un certain nombre de tâches, une partie étant "invisible " mais surtout la prise en charge de toutes les coordinations entre les différents temps et les différents espaces sociaux) est en train de s'estomper et que les difficultés concrètes de la conciliation entre vie professionnelle et vie familiale devraient concerner désormais un grand nombre d'individus. Ce qui signifie aussi, que le plein emploi des Trente glorieuses n'est plus possible "parce qu'il reposait en grande partie sur le modèle de l'homme seul soutien de famille" ${ }^{4}$ (...)

\footnotetext{
${ }^{4}$ JC Barbier, J. Gauthié, Les politiques de l'emploi en Europe et aux Etats-Unis, PUF, 1998
} 